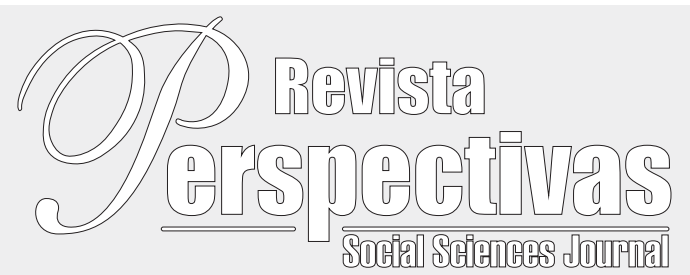

Artículo Original

https://doi.org/10.22463/25909215.2829

\title{
Testimonios de guerra y paz. Una mirada desde las escuelas de la región del Catatumbo
}

Testimonies of war and peace. A view from the schools of the catatumbo region

Maricela Martínez-Guerrero' ${ }^{1}$, Diana Graciela Rojas-Sánchez², Daniel Villamizar-Jaimes ${ }^{3}$

'Licenciada en Educación Preescolar, Centro Educativo Rural el Chamizón, El Carmen Norte de Santander, Colombia. 3214043986. E-mail: maricelamg@ufps.edu.co 2Licenciada en Educación Básica, Centro Educativo Rural Santa Inés, El Carmen Norte de Santander, Colombia.3133404348. E-mail: dianagracielars@ufps.edu.co

${ }^{3}$ Magister en Psicopedagogía Clínica, Departamento de Ciencias Sociales, Humanas e Idiomas, Facultad de Educación, Artes y Humanidades, Cúcuta, Colombia. 3163938305. E-mail: danielvj@ufps.edu.co

Cómo citar: M. Martínez-Guerrero, D.G. Rojas-Sánchez, D. Villamizar-Jaimes “Testimonios de guerra y paz. una mirada desde las escuelas de la región del catatumbo". Perspectivas, vol. 5, no. 2, 44-57.

Recibido:February 05, 2020; Aprobado: May 06, 2020.

\section{RESUMEN}

\section{Palabras claves:}

Conflicto armado, actores educativos, tipos de violencia, región del Catatumbo.
En Colombia el conflicto armado latente se ha caracterizado por las distintas formas de violencia, con particularidades propias de tiempo, contextos, actores y dinámicas que lo hacen aún más complejo y con una característica prominente, respecto a la vulneración de la dignidad y los derechos humanos especialmente contra la población civil. La Región del Catatumbo y sus habitantes han sufrido de cerca los efectos que ha dejado más de cinco décadas de violencia y que se espera que tras la firma del tratado de paz con el grupo guerrillero que tenía mayor influencia en la región, la situación hubiese cambiado para mejorar. Esta investigación recolecta las opiniones de un grupo de estudiantes pertenecientes a la Sede Filo de Palo, y docentes que laboran en la comunidad. Se desarrolla una investigación mixta en la que se utilizan la encuesta y la entrevista como instrumentos de recolección de datos. Los resultados permiten evidenciar en opinión de los informantes las afectaciones han sido muchas tanto a nivel individual como en la comunidad. En ellos se observa cierto pesimismo hacía un mejor futuro y se concluye que el proceso educativo es uno de los sectores que demanda mayor apoyo por parte de los entes territoriales para así ofrecer nuevas y mejores oportunidades de las que hoy día experimentan la comunidad académica

\begin{tabular}{ll}
\hline ABSTRACT \\
\hline Keywords: & $\begin{array}{l}\text { In Colombia the latent armed conflict has been characterized by different forms of violence, with particularities } \\
\text { of time, contexts, actors and dynamics that make it even more complex and with a prominent characteristic, } \\
\text { regarding the violation of dignity and human rights especially against the civilian population. The Catatumbo }\end{array}$ \\
Armed conflict, & $\begin{array}{l}\text { Region and its inhabitants have closely suffered the effects of more than five decades of violence and it is hoped } \\
\text { that after the signing of the peace treaty with the guerrilla group that had the greatest influence in the region, the } \\
\text { educational actors, } \\
\text { types of violence, }\end{array}$ \\
satatumbo region. & to the Filo de Palo Headquarters, and teachers working in the community. A mixed research is developed in \\
& which the survey and the interview are used as data collection instruments. The results show that the informants \\
think that there have been many effects both at individual and community level. They show a certain pessimism \\
towards a better future and conclude that the educational process is one of the sectors that demands greater \\
support from local authorities in order to offer new and better opportunities than those currently experienced \\
by the academic community.
\end{tabular}

\section{Introducción}

El conflicto interno que aproximadamente lleva más de 50 años presente en el territorio colombiano, en elcual sehan involucrado diversos actores estatales y no estatales, ha ocasionado 4.210 masacres, las cuales han dejado 24.447 víctimas mortales y una exuberante cifra de personas desaparecidas desde el año 1.958 hasta el 2.018 según cifras del Centro
Nacional de Memoria Histórica (2.014). Además, otro resultado de la violencia en el país es el número en ascenso de personas desplazadas, mutiladas, torturadas y secuestradas; de las cuales no hay distinción entre hombres y mujeres, niños, jóvenes y adultos. Al mismo tiempo que muchas hectáreas de tierras abandonas y despojadas. 
El conflicto armado es uno de los escenarios que marca de manera más profunda a los individuos y a las sociedades. La irracionalidad y deshumanización de la guerra expone a las personas y a las comunidades a situaciones límite de violencia que generan en ellos procesos de deconstrucción y ruptura del tejido social, de pérdida del bienestar emocional y destrucción de sus proyectos de vida (Nación, 2009, citado por Toro, 2010, p. 8).

Las diversas acciones violentas y agresivas ejecutadas en contra de la población civil dejan entrever los niveles de degradación del conflicto, puesto que las tácticas, estrategias e instrumentos utilizados trasgreden la dignidad y los derechos humanos de las personas, según el Registro Único de Victimas (RUV), a 1 de febrero de 2020 se han registrado 8.989.570 personas como víctimas del conflicto armado en Colombia. Por consiguiente, los daños y afectaciones ocasionados específicamente aquellos intangibles e invisibles a simple vista, desencadenan modificaciones en las realidades de las personas, pese a las adversidades que han debido enfrentar las víctimas.

Las modalidades y prácticas violentas sufridas por miles de personas en Colombia, provocan daños e impactos que afectan la integridad de las víctimas. Los impactos psicológicos deterioran las relaciones interpersonales y la salud física; las pérdidas económicas generan inestabilidad emocional; los impactos colectivos y el daño a las redes sociales y comunitarias afectan las capacidades y posibilidades individuales. Así se configuran un entramado de situaciones, que se relacionan mutuamente, lo cual hace difícil separar y especificar aquello que es propio de cada tipo de daño. (GMH, 2013, citado por Anacona, 2014, p. 13).

La necesidad de comprender las particularidades de las afectaciones según los contextos, actores y dinámicas, logran identificar y reconocer los recursos individuales, familiares y comunitario que las victimas utilizan para tramitar el dolor y enfrentar el sufrimiento de las marcas de la guerra. Es por esto que a través de la narración las victimas encuentran un medio para poder contar su testimonio, ser escuchados y no seguir siendo invisibilizados o reducidos como los daños colaterales que ha producido la guerra en el país. Como lo afirma Tovar (2016): "la narración es vista como catalizadora en tanto que puede favorecer y promover el proceso de recordar y sanar, de revivir a nivel social y de encontrarse con sus sentimientos" (p.45). Es un entramado de relatos con fuertes implicaciones en el presente desde la perspectiva emocional.

El Ministerio de Educación Nacional (MinEducación) ha emanado diferentes artículos y referentes conceptuales que hacen parte de una política de inclusión educativa en la que se busca dar respuesta a la crisis derivada de este importante fenómeno, y en ella se plantea que:

Uno de los desafíos en materia de atención educativa es que el Estado garantice el acceso al servicio público educativo, así como la permanencia en él, tanto de los niños y niñas como de los jóvenes y adultos, sin distinciones de raza, género, ideología, religión o condición socioeconómica (Fundación Saldarriaga Concha, 2015, p.3).

Este trabajo investigativo hace énfasis en las situaciones de conflicto armado que permean e inciden en las instituciones educativas situadas en la región del Catatumbo - Norte de Santander, partiendo de la historia violenta y dolorosa que ha generado el conflicto armado en Colombia. Se pretende abordar esta problemática desde los actores del proceso educativo y las mismas prácticas pedagógicas, ya que son propias del contexto y del día a día de un territorio que al igual que mucho ha sido azotado por los diversos actores del conflicto. En el escenario educativo nacional y de manera específica en las instituciones educativas de la región del Catatumbo, reinciden de forma sistemática las problemáticas propias de un país en crisis: los brotes de violencia propios del conflicto armado, que dejan como 
efecto directo la injusticia social, y secuelas como el desplazamiento forzado son hechos con los que se enfrentan a diario las comunidades educativas.

La cantidad acreciente de niños y niñas víctimas del conflicto armado que ingresan continuamente a las instituciones educativas de la Zona del Catatumbo, se convierten en motivo de preocupación para los docentes, ya que conlleva a arduas condiciones como la desestructuración brusca de la vida cotidiana, por ausencia de casi todos los referentes habituales, y la inseguridad absoluta sobre el futuro. El trabajo, el hábitat, los amigos, la familia, incluso la integridad física y la vida se tornan, por la posibilidad de su pérdida, en frágiles e inciertos. Las creencias y costumbres son puestas en cuestión y la supervivencia es la máxima prioridad (Ibáñez y Díaz, 2010, citado por Toro, 2010, p. 14).

Las instituciones educativas de esta parte del país no cuentan con las herramientas que les facilite abordar con responsabilidad estas situaciones, las prácticas pedagógicas que son una alternativa en la búsqueda de mecanismos para que los docentes puedan conocer las historias de quienes tuvieron que vivir el flagelo de la violencia.

Hoy día se afirma que Colombia está experimentando la fase del posconflicto, en dónde las instituciones educativas y su personal docente no cuentan con los insumos necesarios en sus Proyectos Educativos Institucionales, Manuales de Convivencia, los Planes de estudio, entre otros; no han sido capacitados ni mucho menos reorientados con modelos pedagógicos que faciliten la reconciliación, para que logren un equilibro físico, mental y emocional, además del fortalecimiento de valores como la justicia, la democracia, la tolerancia, la convivencia, el respeto, la cooperación, la autonomía, la racionalidad, el amor a la verdad y la solidaridad, entre una larga lista de necesidades.

También es importante mencionar que el posconflicto ha abierto las puertas a la esperanza y así mismo, a la incertidumbre, característica que refuerza mucho más la importancia de una política educativa fuerte que brinde las herramientas necesarias e indispensables y que posibilite la transformación de la situación. Comprender las lógicas de la transición social y política deseada. La tarea de los docentes del Catatumbo es un poco compleja, lo que la convierte en un reto enorme, pero esperanzador, puesto que se ve la necesidad de transformar el panorama que implica un nuevo sujeto, lleva a plantear la práctica docente en torno a una pedagogía problematizada, en la que el docente articula sus actividades, con las situaciones de la realidad social que incluyen a sus estudiantes y las intencionalidades de cimentar, como establece la ley, hacia una cultura para la paz.

En un informe de la Unidad de Víctimas (2020) deja ver las estadísticas asociadas con el desplazamiento de familias y/o habitantes, que a pesar de los esfuerzos que ha hecho el gobierno mediante los acuerdos para logar la paz, la reconciliación en todo su territorio, estos no han sido suficientes, pues estos solo han quedado en la firma y en las mesas de negociación. En estas comunidades poco ha mejorado las condiciones de vida, puesto que aún persisten los problemas sociales; tal como se establece en el informe Catatumbo, memorias de vida y dignidad publicado por el Centro de Memoria Histórica (2014) la desigualdad, la pobreza, la falta de oportunidades, el desempleo, la falta de garantías para la vida y la salud, el monocultivo de coca, son factores que conllevan a las personas a cometer actos ilegales, a la destrucción y a la muerte.

En región del Catatumbo las instituciones educativas, se ven sumergidas en diversas problemáticas que hacen necesario un manejo diferente en el aula. Por un lado, se enfrenan del ingreso durante todo el año escolar de niños y niñas en situación víctimas del conflicto, que obliga a los maestros atender una serie de dificultades en el aula de clase debido a la situación particular de cada estudiante, destacando las diferencias en las edades entre los estudiantes regulares y los estudiantes 
víctimas, que sumado a los efectos traumáticos que experimentan estos estudiantes por las experiencias vividas.

Por otra parte, se agudiza la situación en las aulas al aumento en la cifra de niños y niñas venezolanos según estadísticas del Alto Comisionado de las Naciones Unidas para los Refugiados (ACNUR) señalan que hay 327.000 niños y niñas venezolanos en el país que llegan a las instituciones educativas con condiciones académicas diversas que generan un mayor esfuerzo y compromiso por parte de los maestros. Esta problemática presiona y resalta la necesidad del enfoque educativo inclusivo.

En este sentido la UNESCO define la educación inclusiva en su documento conceptual (i) así:

La inclusión se ve como el proceso de identificar y responder a la diversidad de las necesidades de todos los estudiantes a través de la mayor participación en el aprendizaje, las culturas y las comunidades, y reduciendo la exclusión en la educación. Involucra cambios y modificaciones en contenidos, aproximaciones, estructuras y estrategias, con una visión común que incluye a todos los niño/as del rango de edad apropiado y la convicción de que es la responsabilidad del sistema regular, educar a todos los niño/as.

De acuerdo con lo anterior se requiere un mayor esfuerzo que permita garantizar la inclusión educativa a la víctima del conflicto a partir de herramientas pedagógicas apropiadas y contextualizadas a las necesidades del entorno. Por esta razón, es importante conocer las percepciones y vivencias de los actores educativos de la región del Catatumbo sobre los efectos que ha tenido la guerra en sus vidas y sobre las expectativas que se tienen sobre el proceso de paz en la región.

Narrativas como alternativa en niños y niñas víctimas del conflicto. Cabe destacar que las experiencias de vida influyen tanto en la forma de dar significado y concebir un suceso relevante, como en la manera de narrarlo, es así, que las personas víctimas del conflicto destacan las afectaciones de diversas índoles que han trascurrido en sus vidas y como ha sido el proceso para superar esta situación que de alguna manera las diferentes situaciones por las que han tenido que pasar no han sido menos dolorosos, para poder empezar de nuevo, unos con una mejor vida y otros que aún siguen esperanzados en que sus vidas algún día volverán a ser las mismas de antes.

Con el fin de abordar el tema sobre el asunto de las narrativas como alternativa en niños víctimas del conflicto, esta investigación se apoya en texto Pensar desde la Infancia desde América Latina de Otálora (2019), el cual se centra en la construcción de los niños como sujetos políticos en contextos de conflicto armado. Partiendo de la idea, que las interacciones en dichos contextos se dan a través de las prácticas dialógicas y que estas son las que cobran sentido en la cultura, en el tiempo, en el espacio y en la sociedad.

De lo anterior, se puede considerar que según (Otálora, 2019) "La condición de infancia en contexto de conflicto armado se constituye a partir de las voces presentes en los relatos de los distintos actores que participan del conflicto" (p. 58).

Por consiguiente, los niños víctimas del conflicto no solo generan una forma de pensamiento, sino que, además, esta situación se caracteriza por las afectaciones que acarrea en el niño por las huellas de la violencia, suscitando concepciones de niñez dentro del conflicto, donde los niños y las niñas dejan de ser sujetos de derechos para ser un medio de legitimización de las dinámicas de la guerra.

Por lo tanto, la comprensión de los significados se da a partir de procesos mediados por el lenguaje y las relaciones en los diferentes escenarios en que se desenvuelven los niños y las niñas en esta condiciones, permite la construcción de sus subjetividades y la comprensión de su realidad en cada una de las experiencias significativas donde quiera que participan, de igual manera algunos de 
los casos de estos niños y niñas han demostrado que son fortalecidos y muestran el desarrollo de sus potencialidades, reconociendo que este hecho los ha vuelto valientes y con ímpetu de emprender nuevos proyectos.

Debido a esta situación y a las diferentes causalidades que han definido a la guerra como un sinfín, en el que la niñez muchas veces ha sido protagonista directa e indirectamente, se han desbordado una serie de consecuencias que agitan sus diferentes contextos generando algunas afectaciones psicológicas, físicas y emocionales, pero que paradójicamente hay quienes se han constituido en interacciones con otros actores sociales, basándose en la naturalización de diversas violencias a partir del conflicto armado y muchos de ellos logran reconfigurar sus sentidos y prácticas a partir de construcciones colectivas.

A través de las narraciones los integrantes de las familias que llevan un tiempo de asentamiento corto, y particularmente los desplazados, por el miedo o la amenaza, suelen desarrollar reflexiones en torno a los relatos y en los que, al exaltar el valor fundamental de la vida, le dan un sentido a su experiencia. Este hecho es fundamental en el proceso de asumir el cambio y reorganizar la familia bajo las nuevas circunstancias (Córdoba Jaramillo \& Santa Tobón, 2016).

De esta manera es importante recalcar que la narración es un elemento importante para crear otras formas de resignificar. A través de los relatos se da sentido y significado a las experiencias de las personas, en este caso en específico se visibilizan las narrativas de los individuos víctimas del conflicto, convirtiéndose en una herramienta fundamental para que se logre expresar el dolor, el sentimiento, el miedo y la falta de oportunidades para lograr una mejor vida. Además, sus historias de vida mediante la narración se pueden evidenciar alguna capacidad de agenciamiento y una construcción social sobre posibles opciones alternativas a la violencia.
Niños y niñas en condición de desplazamiento. El conflicto armado existente en el país, ha utilizado diversas formas de proporcionar miedo y dolor en el territorio, en este caso específico se hará mención al desplazamiento forzado, que como lo refiere Tovar (2016)

Es una expresión del conflicto armado que presenta de forma evidente repercusiones individuales y sociales y que más allá de una problemática política o económica se convierte en una tragedia humanitaria que exige la intervención de todos los sectores de la sociedad interesados en la superación de la crisis nacional (p. 72).

La imagen de sí mismos que las personas víctimas del desplazamiento (individual y colectivo) han construido históricamente y que les ha permitido diferenciarse o distinguirse de otros, y al mismo tiempo ser reconocidos por otros, es desestructurada y reconstruida a la luz de las nuevas realidades y posiciones sociales que están obligados a asumir (Bello, 2002, p. 112).

La infancia no ha sido ajena de esta realidad, ya que en una gran proporción la población desplazada principalmente de sectores rurales son familias completas que tienen que abandonar sus hogares en busca de nuevas oportunidades y opciones de vida, es así como los niños y niñas que se encuentran en un proceso de construcción de subjetividades y aprehensión de su realidad objetiva, modifican sus condiciones de vida en los diferentes contextos donde interactúan afectando así sus relaciones sociales.

Los niños víctimas del conflicto han sido protagonistas de unas etapas muy difíciles de su vida bien sea de manera directa e indirecta, los flagelos del conflicto por lo general permean la vida de los menores, en el caso de los niños desplazados, esta situación genera secuelas que marcan notoriamente la vida de quienes les tocó vivir estos sucesos, como situaciones de soledad, resentimientos, afectaciones psicológicas, miedos, zozobras, venganzas, 
abandonos y desconfianza frente a un mundo predecible. De igual manera hay quienes por medio de este conflicto y su interacción permanente con el mismo, han naturalizado los hechos y violencias dentro de sus prácticas cotidianas.

La construcción social del niño o joven se hace a través de la relación que tienen con el otro, con sus familias, con sus vecinos, con sus pares y con los valores que son socialmente construidos por las costumbres y la cultura. Todos estos aspectos y muchos más son modificados, influidos, trastocados, y tergiversados por el conflicto armado. La cotidianidad de la guerra hace que los jóvenes construyan imaginarios y formas de relación mediado por los valores y símbolos propios de la guerra. Los símbolos que estas personas representan están sustentados en el poder, en la vida fácil (Andrade Salazar, 2010).

Las secuelas del conflicto armado son situaciones muy difíciles para los menores de edad, ya que al estar involucrados directamente en estas situaciones que generan odios, guerra y venganzas. Las cicatrices dejadas por los hechos desgarradores vivenciados, los convierten en testigos, que en ocasiones son apuntados como objetivos de persecución, torturas, desplazamientos, violencia sexual y muerte; inclusive estas atrocidades llegan ser perpetuadas en comunidades enteras.

El rostro de la guerra sigue siendo joven pues envuelve a la niñez y la juventud en esta realidad violenta e inhumana, mostrándoles miedo, dolor, pobreza y muerte. De ahí, surge la necesidad de actuar porque el futuro del país no puede seguir envuelto en la violencia como se está viendo comprometido esté presente. Hay que mencionar, que los escenarios educativos no son ajenos a los efectos de la guerra, ya que estos espacios a veces son blancos de ataques, resultando vulnerados en sus diversas dimensiones estructura física, coacciones contra los estamentos (administrativos, docentes y estudiantes), currículos descontextualizados a las problemáticas latentes de la realidad.

Por tal motivo las instituciones educativas como actor predominante en el proceso de educación formal, recibe múltiples afectaciones por el conflicto y sus secuelas como el desplazamiento forzado.

Teniendo en cuenta a Bello (2002) la reconstrucción de la memoria de las personas que han sufridos hechos victimizantes, hechos como el desplazamiento; es un medio reparador de las secuelas de los sucesos traumáticos y además una oportunidad de direccionarse hacia el camino de la resiliencia.

Victimas. Según las Naciones Unidas (ONU) Derechos Humanos (1985) Se entenderá por víctimas las personas que, individual o colectivamente, hayan sufrido daños, inclusive lesiones físicas o mentales, sufrimiento emocional, pérdida financiera o menoscabo sustancial de los derechos fundamentales, como consecuencia de acciones $u$ omisiones que violen la legislación penal vigente en los Estados Miembros, incluida la que proscribe el abuso de poder (p. 1).

Al hacer mención a la palabra víctimas, es necesario hacer referencia que no solo el conflicto armado victimiza a las personas, también la destrucción de los hogares, el abandono, la falta de valores y normas en la sociedad conlleva a que muchos casos de personas que han sido intimidados y violentados sus derechos fundamentales, incluso el mismo Estado por las pocas oportunidades que tienen los jóvenes y las personas de escasos recursos económicos que hoy están siendo víctimas del flagelo del desempleo, entre otros.

En la región del Catatumbo se ven miles de personas que han sido afectados y violentados sus derechos por diferentes actores del conflicto armado en Colombia, familias enteras que han tenido que abandonar sus tierras, sus familias, en busca de 
proteger sus vidas; pero cuando llegan en busca de atención por las unidades de víctimas, también se convierten en otras víctimas del Estado, porque para poder recuperar sus derechos fundamentales es casi imposible que el mismo le brinde garantías por la cantidad de requisitos que esto conlleva.

\section{Materiales y métodos}

\section{Características de la investigación}

Para el desarrollo de esta investigación se asume un paradigma mixto, en el cual se interrelacionan y complementan las fortalezas de los enfoques cuantitativo y cualitativo, tal como lo menciona Gómez-Juncal, Pérez-Fabello \& Campos (2009), las investigaciones mixtas permiten "la inclusión de opiniones, recolección de datos y técnicas de análisis e inferencias de ambos enfoques, con el fin de ampliar y profundizar la comprensión y corroboración" (p.11). Desde esta perspectiva, se pretende un mejor entendimiento de la problemática abordada puesto que se complementan las fortalezas del paradigma cuantitativo (descriptivos, tendencias e inferencias) y del cualitativo (en cuanto al nivel de profundidad y entendimiento que se alcanza del contexto).

El nivel de la investigación hace referencia al grado de profundidad con que se aborda un fenómeno objeto de estudio (Arias, 2012). Por ende, se puede afirmar que esta investigación aborda el problema desde el nivel descriptivo, ya que se pretende caracterizar el efecto que ha dejado las acciones violentas tanto en docentes como en estudiantes de la región del Catatumbo, específicamente en Centro Educativo Rural El Chamizón en su Sede Filo de Palo, tal como se define "la investigación descriptiva consiste en la caracterización de un hecho, fenómeno, individuo o grupo con el fin de establecer su estructura o comportamiento" (Arias, 2012, p. 24).

El diseño de la investigación es la estrategia general que adopta el investigador para responder al problema planteado, es decir, está directamente asociado con la forma como se espera recolectar los datos. A partir de la definición anterior, esta es una investigación de campo ya que los datos son recolectados directamente de los sujetos objeto de estudio.

\section{Población y muestra}

Para Arias (2012) la población es "un conjunto finito o infinito de elementos con características comunes para los cuales serán extensivas las conclusiones de la investigación” (p. 81). La población del presente estudio está conformada por los estudiantes de quince diferentes sedes de instituciones educativas ubicadas en los diversos municipios de la Región del Catatumbo - Norte de Santander.

En esta investigación se pretende caracterizar las opiniones de la totalidad de estudiantes (16) de la Sede Filo de Palo del Centro Educativo Rural El Chamizón, por ende, se aplica en un primer momento el muestreo no probabilístico para seleccionar este centro educativo y luego en un segundo momento se aplica un censo puesto que todos los estudiantes del centro educativo de una u otra forma se han visto afectados por el conflicto armado en algún momento de sus vidas. Las características demográficas de los estudiantes informantes son las siguientes: el $75 \%$ son mujeres y el $25 \%$ hombres, con edades que oscilan entre 6 y 13 años concentrando el 63\% de los casos en el rango de 7 y 9 años. En lo que respecta al grado que cursan, el $75 \%$ de los estudiantes se distribuyen proporcionalmente entre los grados Tercero, Cuarto y Quinto. En la totalidad de los casos los estudiantes viven con sus padres y hermanos, como principal característica de su composición familiar.

En lo concerniente al segundo grupo de informantes, correspondiente a los docentes. Se recolectó la opinión de seis docentes cada uno a cargo de una sede educativa que funciona en la región bajo el mismo modelo educativo Escuela Nueva, luego en este caso se recurre a un muestreo no probabilístico por conveniencia, puesto que el criterio de inclusión era el que los informantes accedieran a proporcionar 
información. Este grupo de docentes se caracteriza por tener diversidad de formación profesional, con predominio del género femenino y de la formación posgradual a nivel de especialización. Al menos el $60 \%$ son casado y llevan laborando como mínimo tres años en la región bajo la modalidad de vinculación de contrato término fijo o en condición de provisionalidad.

\section{Instrumentos de recolección de datos y su validación}

Para caracterizar el nivel de afectación del informante se adaptó el diseño de la Matriz de Identificación de Daños que da el Centro Nacional de Memoria Histórica - CNMH (citado en GalloFlórez y Martínez-Balaguera, 2020) como se muestra en la Tabla I, en la se presenta la caracterización de la afectación en función del tipo de daño sufrido, a partir de la formulación de una serie de preguntas que han sido incluidas en el cuestionario que se aplicó a los dos grupos de informantes.

\begin{tabular}{|c|c|}
\hline \multicolumn{2}{|c|}{ de paz } \\
\hline DIMENSIÓN & INDICADORES \\
\hline Daños Morales & Ítems $3,4,18,19,20$ \\
\hline $\begin{array}{l}\text { Daños Psicológicos } \\
\text { emocionales }\end{array}$ & Ítems $5,6,10,16,21,22,23$ \\
\hline Daños Socioculturales & Ítems 25,26 \\
\hline Daños Ambientales y Materiales & Ítems $17,27,11,12$ \\
\hline Daños a la Familia & Ítems $13,14,15,29,30$ \\
\hline Daños al Proyecto de Vida & Ítems $7,8,9,24,28$ \\
\hline Proceso de Paz & $\begin{array}{l}\text { Ítems } 31,32,33,34,35,36,37 \text {, } \\
38,39\end{array}$ \\
\hline
\end{tabular}

Fuente. Gallo-Flórez y Martínez-Balaguera, y Autores

Tras la aplicación estadística de la prueba de confiabilidad (Alfa de Cronbach) se logró determinar un coeficiente de 0,749 que, según Cohen \& Swerdlik (2001), Fuentealba (2006), Prieto \& Delgado (2010) presenta una confiabilidad válida para estudios sociales. Así mismo, se determinó el índice de homogeneidad corregido o también llamado índice de discriminación del instrumento. Se pudo verificar que todos los ítems discriminan muy bien puesto su valor ID $\geq 0,40$ (Muñiz, 2005); a excepción del ítem 23 que se decide mantener en el cuestionario por la temática que aborda y que está directamente asociada con la investigación.

\section{Resultados}

Los resultados se presentan en función del tipo de informante, es decir, primero los estudiantes y luego los docentes.

3.1. Percepción de los Estudiantes sobe las afectaciones derivadas del conflicto

Como primer elemento a resaltar es que la totalidad de los estudiantes encuestados de la Sede Filo de Palo del Centro Educativo Rural El Chamizón afirman que se sienten víctimas del conflicto armado, resaltando el hostigamiento (en cuatro de cada cinco estudiantes) y el desplazamiento forzado como los principales hechos victimizantes por parte de los grupos al margen de la ley (Salazar, Barranco, Ruiz, Marín \& González, 2016).

Daños Morales. El 93,8\% de los estudiantes afirman que han sufrido señalamientos o discriminación por su condición de víctima en el contexto social en dónde viven. Mientras que el $87,5 \%$ afirman que eventualmente experimentaron sentimientos de humillación o maltrato durante el desarrollo del evento violento que experimentaron, pero en su totalidad afirman que no sienten vergüenza por ello (Correa, 2020). Se resalta como principal afectación en esta categoría, que en opinión del 93,8\% de los estudiantes los sentimientos de desolación y tristeza siempre están presentes en su vida como efecto de la ausencia de familiares o amigos que han sido víctimas del conflicto armado que se vive en la región.

Daños Psicológicos y Emocionales. El 93,8\% de los encuestados afirman que en sus mentes persisten los recuerdos angustiantes del momento en que ocurrieron los hechos violentos, situación que los lleva constantemente a experimentar miedo y nerviosismo. Al indagar sobre los sentimientos que 
experimentan por la ausencia de familiares a causa de los eventos violentos, el $81,3 \%$ reconocen que la desolación y la tristeza son sentimientos recurrentes en sus vidas. Se resalta como principal efecto, que el $75,1 \%$ de manifiestan dificultades en la interacción con otras personas y en el establecimiento de metas a través del tiempo, puesto que consideran que su futuro es muy incierto (Balcázar, Mejía 6 Muñoz, 2014).

Daños Socioculturales. Al indagar sobre las afectaciones sociales que han experimentado los informantes como víctimas de los hechos violentos derivados del conflicto armado que se vive en la Región del Catatumbo, se pudo ratificar que el 93,8\% de ellos aseguran les han generados señalamientos en el contexto social donde residen lo que ha afectado las relaciones entre los miembros de la comunidad (Gómez Pimienta \& Grueso-Hinestroza, 2019).

Daños Ambientales y Materiales. Cuando se les preguntó a los informantes, sobre las prácticas realizadas por los victimarios que tenían afectación directa en el medio ambiente o en el contexto en donde residían, la totalidad de ellos coincidieron en reconocer que las voladuras del oleoducto Caño Limón - Coveñas es el acto terrorista con mayor afectación al medio ambiente, puesto que por medio de este tipo de acciones se contaminan los suelos y los ríos, que en muchos casos es el sustento para la agricultura y el consumo humano (Ibáñez \& Díaz, 2010).

Daños a la Familia. Al indagar en los informantes las afectaciones familiares a causa de los hechos violentos de los que han sido objeto, se determinó que la totalidad de ellos reconocen que su estructura familiar no se ha visto afectada (salvo aquellos casos asociados a desapariciones forzosas) pero si se han evidenciado cambios de roles en el $93,7 \%$ de los casos. Asimismo, afirman que hoy día después de esas acciones violentas, se han fortalecido las relaciones familiares las cuales se fundamentan en sentimientos de respeto, diálogo, solidaridad y unidad. En dónde si reconocen que se han presentado afectaciones, es en los planes y metas familiares puesto que muchas de ellas se fundamentaban en actividades agrícolas.

Daños al Proyecto de Vida. Se puede determinar que el $68,8 \%$ de los informantes han presentado afectaciones emocionales tras el acto violento, lo que ha producido modificaciones en el desarrollo de sus actividades diarias. En los estudiantes informantes se evidencia una postura de resiliencia puesto que, a pesar de los hechos vividos, el 93,7\% de ellos afirman que han tratado de dejar esta situación en el pasado para poder continuar con su proyecto de vida, con la menor afectación posible.

Finalmente, la totalidad de los estudiantes reconocen que a nivel familiar la violencia propició cambios de residencia o de actividad laboral o en el peor de los casos, redujo las oportunidades a las que inicialmente podían tener acceso (González Gil, 2010; Migraciones, 2013).

\subsection{Percepción de los Docentes sobe las} afectaciones derivadas del conflicto

Al igual que lo afirmado por los estudiantes, la totalidad de los docentes consideran que de una $\mathrm{u}$ otra forma han sido afectados o se consideran víctimas del conflicto armado que predomina en esta región del país (Quiceno, 2013; Ríos, 2016).

Daños Morales. El 66,7\% de los docentes afirman que no han sufrido señalamientos o discriminación por su condición de víctima en el contexto social en dónde viven. Pero las opiniones entre los docentes están divididas, pues la mitad de ellos afirman que eventualmente sintieron humillación o maltrato durante el desarrollo del evento violento que experimentaron, mientras que la otra mitad afirman que sienten vergüenza por los que les ha tocado vivir en el marco del conflicto interno que se vive en nuestro país. En lo que, si coinciden todos es en afirmar que, ante la ausencia de un ser querido, la desolación y tristeza se han apoderado de sus vidas. 
Daños Psicológicos y Emocionales. la totalidad de los docentes encuestados aseguran que posteriormente a los hechos victimizantes el miedo que sienten se manifiesta por medio de nerviosismo y constante estado de alerta por los eventos que podrían repetirse. El 33,3\% aseguran que han intentado continuar con la cotidianidad de sus vidas, pero les resulta muy difícil recuperar la normalidad. Finalmente, la mitad de ellos manifiestan dificultades en la interacción con otras personas y en el establecimiento de metas a través del tiempo.

Daños Socioculturales. Dentro de las afectaciones sociales que han experimentado los docentes como víctimas de los hechos violentos derivados del conflicto armado que se vive en la Región del Catatumbo, se pudo ratificar que el 66,7\% de ellos aseguran les han generados señalamientos en el contexto social fuera de la comunidad en donde residen lo que ha afectado las relaciones entre los miembros de la misma (Olaya, 2015).

Daños Ambientales y Materiales. Cuando se les preguntó a los docentes, sobre las prácticas realizadas por los victimarios que tenían afectación directa en el medio ambiente o en el contexto en donde residían, el $83,3 \%$ de ellos coincidieron en reconocer que los cultivos ilícitos son el principal agente generador de afectación al ambiente por la deforestación que se genera con el fin de ampliar las zonas de cultivo, mientras que el porcentaje restante menciona las voladuras del oleoducto Caño Limón - Coveñas.

De forma complementaria, los docentes afirman que el desplazamiento forzado, sumado a la explotación ilegal de recursos mineros, a la tala indiscriminada de árboles para la obtención de tierras que posteriormente son destinadas a cultivos ilícitos y las constantes voladuras del oleoducto Caño Limón - Coveñas han producido desarraigo de las costumbres propias de la región en la mitad de los docentes encuestados, el resto de las personas afirman que se han mantenido resistentes a pesar de las situaciones adversas.
Daños a la Familia. Se determinó que el 66,7\% de los casos no se ha visto afectada la estructura familiar, mientras que el porcentaje restante si sufrió cambios ante la desaparición y/o muerte de su pareja sentimental, situación que llevó al $50 \%$ de ellos a asumir nuevos roles dentro de las dinámicas familiares. Asimismo, el 83,3\% de los docentes encuestados aseguran que después de los acontecimientos violentos de los que fueron víctimas, al interior en sus hogares las relaciones son muy buenas y se caracterizan por estar fundamentadas en el respeto, la unidad y el diálogo en busca de soluciones a las situaciones que ofrece el día a día. Con relación a la transformación de planes y metas familiares como efecto del hecho violento acontecido, la mitad de los docentes afirman que si han sufrido cambios.

Daños al Proyecto de Vida. El 66,6\% de los docentes se han visto afectados en el campo emocional como resultado del acto violento, lo que ha producido modificaciones en el desarrollo de sus actividades diarias, resaltando que en el 33,3\% de ellos se observan estas dos condiciones de afectación.

En lo pertinente a la resiliencia que poseen los docentes encuestados, se pudo determinar que la mitad de ellos han intentado continuar con sus vidas a pesar de los acontecimientos vividos, alcanzando avances significativos en este proceso. Para finalizar, el 50,0\% de ellos reconocen que a nivel familiar la violencia los llevó a adoptar cambios en diversos aspectos de sus vidas que de una u otra forma han incidido en su evolución como persona y profesional.

3.3. Percepción de los Estudiantes sobre el Proceso de Paz y sus efectos en la Región

Al indagar entre los informantes estudiantes del Centro Educativo Rural El Chamizón, sobre su percepción del Proceso de Paz adelantado entre el Gobierno y el grupo guerrillero de las Fuerzas Armadas Revolucionarias de Colombia - FARC, el cual ha tenido injerencia en la región del Catatumbo por más de cuarenta años. Con la llegada de las Autodefensas Unidas de Colombia - AUC a 
comienzos del 2000 perdió control sobre la zona, pero posteriormente tras realizar alianzas con las guerrillas del ELN y el EPL, así como con las bandas criminales emergentes entre las que se encuentran Los Rastrojos, las Autodefensas Gaitanistas de Colombia, Los Urabeños o el Clan Úsuga.

Logran en 2015 recuperar el control de la zona, la cual le resulta de vital importancia por diversas razones dentro de las que se destacan: los sembradíos de hoja de coca para uso ilícito, la producción de clorhidrato de cocaína, su exportación a los mercados internacionales por medio de Venezuela, que sumado al contrabando ilegal de gasolina y el poder resguardasen en el vecino país de los controles territoriales que ofrecen las fuerzas del Estado.

A partir de esta introducción al contexto, se indagó con los estudiantes si ellos como residentes de la zona apoyaban el Proceso de Paz, a lo cual todos manifestaron estar de acuerdo. Pero cuando se explora sobre los cambios que se han experimentado en la región tras la firma del acuerdo con este grupo armado (siendo que eran ellos los que controlaban la región) la totalidad también respondieron que a la fecha no han visto ningún tipo de cambio (Iguaran, 2011). En este sentido, el 53,6\% de ellos afirman que tras la firma del Acuerdo de Paz han aumentado los cultivos ilícitos en la región y el porcentaje restante ratifican la idea que los problemas siguen siendo los mismos y en la misma intensidad.

Dentro de los compromisos pactados en La Habana estaba el proceso de Justicia, Verdad y Reconciliación entre los victimarios y sus víctimas. En este sentido, la totalidad de estudiantes encuestados aseguran que no es posible que se alcance la reconciliación ni el perdón por los hechos acontecidos, puesto que consideran que no hay un proceso de justicia dado que no apoyan la idea de que sus victimarios no paguen cárcel por los crímenes cometidos.

Finalmente, al indagar sobre su aceptación al desarrollo de la Cátedra de la Paz dentro de las instituciones educativas, consideran que es una buena estrategia en el proceso de capacitación a las comunidades en la resolución pacífica de conflictos. Para los encuestados los hechos ya sucedidos no tienen forma de reconciliación, pero manifiestan que el futuro puede ser diferente, siempre y cuando en el país ocurra una transformación que pueda garantizar igualdad de oportunidades para todos en las que se incluya salud y educación gratuita y de calidad para todos los colombianos, sólo así se puede alcanzar la paz.

\subsection{Percepción de los Docentes sobre el} Proceso de Paz y sus efectos en la Región

Tras consultar la opinión de los estudiantes, se complementa este panorama con la opinión de los docentes, a lo cual se inicia preguntándoles sobre su apoyo al Proceso de Paz, pregunta que obtiene un apoyo total en ellos, pero coinciden con sus estudiantes al manifestar que tras la firma del mismo, en la región no se han observado cambios positivos, por el contrario se evidencia que los problemas siguen siendo los mismos y en la misma intensidad, que sumado al aumento de los cultivos ilícitos siguen golpeando fuertemente a los pobladores y generando un estigma de la región a nivel nacional e internacional.

Comoyasemencionó, dentrodelos compromisos pactados en La Habana se esperaba que de parte de los victimarios se diera inicialmente información sobre los hechos acontecidos en la región, resaltando como principal acto de reconciliación conocer el paradero de muchas personas desaparecidas, para así poder recuperar sus restos, darles cristiana sepultura y cerrar ese capítulo del duelo al interior de las familias. En este sentido, la totalidad de docentes encuestados afirman que no es posible que se alcance la reconciliación ni el perdón por los hechos acontecidos, puesto que consideran que no hay un proceso de justicia dado que no apoyan la idea de que sus victimarios no paguen cárcel por los crímenes cometidos, mucho menos de decir la verdad, puesto que ello posiblemente les afecte su libertad. Luego ellos consideran que así es imposible alcanzar la 
reconciliación entre los agentes generadores de violencia y la población civil, que durante más de cinco décadas han vivido toda clase de afectaciones a causa de intereses particulares.

Finalmente, al indagar sobre su aceptación al desarrollo de la Cátedra de la Paz dentro de las instituciones educativas (Jiménez, 2012), las opiniones de los docentes coinciden plenamente con las de sus estudiantes, puesto que reconocen en ella una buena estrategia en el proceso de capacitación a las comunidades tendientes a la búsqueda de medios alternativos de resolución de conflictos diferentes a la de una acción violenta y lesiva. Como ya se mencionó en el párrafo anterior, es imposible alcanzar la paz, si no hay verdad y justicia ante los hechos ya acontecidos; pero para situaciones futuras, consideran necesario que exista igualdad de oportunidades para todos en las que se incluya salud y educación gratuita y de calidad para los colombianos, sólo así se puede alcanzar la paz.

\section{Conclusiones}

Con la realización de esta investigación se identificó la ineficiencia de todas aquellas instituciones del Estado que han sido creadas con el objetivo de apoyar a las víctimas en la implementación de diversos programas que permitan mitigar las afectaciones o secuelas que han dejado los hechos violentos en sus comunidades. Se podría llegar a afirmar que el camino de las víctimas nunca deja de verse afectado puesto que aparte de sufrir el evento violento en sus vidas, terminan siendo revictimizados por la sociedad o por estas instituciones del Estado creadas supuestamente para apoyarlas.

Se determinó que la totalidad de los informantes sienten que han sido víctimas del conflicto armado que se vive en la Región del Catatumbo (Leal, 2019; Loingsigh, 2008). En lo que respecta a las afectaciones que han experimentado los informantes una de las más relevantes es la derivada del desplazamiento forzado, puesto que este evento violento produce desarraigo cultural y personal, teniendo mayor impacto en niños o adolescentes llevando al punto de la pérdida de elementos distintivos propios de su cultura, por lo que se podría afirmar que estos eventos violentos están exterminando una cultura.

Otra afectación derivada de los hechos de conflicto armado y sus secuelas que se hacen presentes en los actores educativos, es el temor y el nerviosismo por los hechos pasados y una constante sensación de incertidumbre por los posibles hechos futuros que afectan la tranquilidad y las dinámicas cotidianas de los participantes.

Estos hechos son una realidad con la que tienen que convivir los habitantes, siendo un gran reto para los docentes que laboran en las diferentes sedes del centro educativo, puesto que, aparte de cumplir con sus deberes académicos exigidos por el Ministerio de Educación Nacional, debe dar orientación y acompañamiento a los estudiantes en la aceptación y superación de estos eventos.

Se evidenció coincidencias en casi la totalidad de las posturas tanto en los estudiantes como en los docentes encuestados, pero en dónde si se observó diferencias marcadas fue al indagar sobre las afectaciones al medio ambiente, en este aspecto los docentes reconocen la tala de bosques para la siembra de cultivos ilícitos como la principal afectación (López Guarin, 2019; Peña, 2013); mientras que los estudiantes afirman que son las voladuras del oleoducto puesto que contamina la tierra y los ríos. Estas diferencias podrían estar generadas por el encubrimiento que los habitantes de la región hacen sobre los cultivos ilícitos, puesto que para ellos es una excelente fuente generadora de recursos económicos.

\section{Referencias}

Anacona. H. M. O. (2014). Revisión documental del concepto "enfoque psicosocial" en atención a víctimas del conflicto armado colombiano particularidades y reflexiones para una acción sin daño. [Tesis de grado]. Universidad Nacional 
de Colombia, Colombia

Andrade Salazar, J. A. (2010). Mujeres, niños y niñas, víctimas mayoritarias del desplazamiento forzado. Revista Cientifica Ciencias Humanas, 6(16), 28-53. Recuperado de: https://www. redalyc.org/pdf/709/70916426003.pdf

Arias, F. G. (2012). El proyecto de investigación. Introducción a la metodología científica. 6ta. Fidias G. Arias Odón.

Balcázar, A., Mejía, K., \& Muñoz, D. (2014). z. [Tesis de grado] Universidad de la Salle. Bogotá.

Bello. M. N. (2002). Narrativas alternativas: medios para reconstruir la identidad. Bogotá.

Gómez-Juncal, R., Pérez-Fabello, M. J., \& Campos, A. (2009). Sujetos bajos en viveza de imagen: efecto del reaprendizaje en la memoria de reconocimiento a corto ya largo plazo con listas mixtas de palabras. Actas do X Congresso Internacional Galego-portugués de Psicopedagogia. Braga: Universidade do Minho. ISBN: 978-972-8746-71-1.

Centro Nacional de Memoria Histórica. (2014) Aportes teóricos y metodológicos para la valoración de los daños causados por la violencia. Bogotá: CNMH.

Cohen, R. \& Swerdlik, M. (2001). Pruebas y Evaluación Psicológicas. 4 ed. México, D.F, México: Editorial Mc Graw Hill.

Córdoba Jaramillo, J. O., \& Santa Tobón, S. M. (2016). Influencia del desplazamiento forzado en los procesos de socialización política que se desarrollan en la escuela (Master's thesis). CONVENIO CINDE - Universidad DE Manizales, Medellín

Correa. G. J. H. (2020). Desarrollo socio afectivo en niños víctimas de conflicto armado colombiano: apego, autorregulación emocional y resiliencia. [Tesis de grado]. Universidad de la Sabana, Colombia

Fundación Saldarriaga Concha. (2015). Hacia una educación inclusiva reto y compromiso de todos en Cundinamarca. Colombia: Departamento de Cundinamarca - Secretaria de Educación. Recuperado de https: www.men.gov.co/978-958691-885-5

Fuentealba, R. G. (2006). El error estándar de medida y la puntuación verdadera de los test psicológicos: Algunas recomendaciones prácticas. Terapia psicológica, 24(2), 117-129.

Gallo-Flórez, M. A. y Martínez-Balaguera, M. (2020). Análisis del daño sufrido por las jóvenes víctimas del conflicto armado de la Institución Educativa Nuestra Señora de las Mercedes, Sardinata. [Tesis de grado]. Universidad Francisco de Paula Santander, Colombia

Gómez Pimienta, L., \& Grueso-Hinestroza, M. P. (2019). Secuelas de la guerra: lo que podemos decir sobre la inclusión laboral de personas en proceso de reintegración. Tendencia Editorial UR.

González Gil, A. (2010). Viajeros de ausencias: desplazamiento forzado y acción colectiva en Colombia (Tesis Doctoral). Universidad Complutense De Madrid, Madrid.

Ibáñez, V., \& Díaz, D. (2010). La respuesta social y comunitaria en las situaciones de guerra y violencia organizada. En P. P. Sales, Actuaciones psicosociales en guerra y violencia política. (pp16-36). Recuperado http://hdl.handle. net/10720/8.

Iguaran. D. J. L. (2011). Narrativas de violencia de las y los jóvenes desvinculados de grupos armados al margen de la ley. [Tesis de grado]. Pontificia Universidad Javeriana, Colombia 
Jiménez, F. (2012). Práctica pedagógica y cultura de paz. Un diálogo necesario. Bogotá, Colombia: ECOE.

Leal, K. L. R. (2019). Representaciones de testimoniantes como víctimas y de la guerrilla de las FARC como victimaria en cuatro relatos del secuestro en Colombia. Revista Ístmica, (24), 67-91.

Loingsigh, G. (2008). Catatumbo: Un reto por la verdad. el Catatumbo. Colombia: Comité de Integración social del Catatumbo

López, M.\& Guarín, C. (2019). Proceso de paz con las Farc y cultivos de coca en el período 2013-2017. (Documento CEDE No. 2019-32, Recuperado de https://ssrn.com/abstract=3449221 or http:// dx.doi.org/10.2139/ssrn.3449221

Migraciones, O. I. (2013). Dinámicas del conflicto armadoenelCatatumboysuimpactohumanitario. USAID. Recuperado de http://cdn.ideaspaz.org/ media/website/document/5b72fe7f2b9d1.pdf, 3 .

Ministerio de Educación Nacional (2009) Decreto No. 1290, Por el cual se reglamenta la evaluación del aprendizaje y promoción de los estudiantes de los niveles de educación básica y media. REPÚBLICA DE COLOMBIA

Muñiz, J. (2005). Análisis de los Ítems. Editorial La Muralla: Madrid: España.

Naciones Unidas, Derechos Humanos. (1985). Declaración sobre los principios fundamentales de justicia para las víctimas de delitos y del abuso de poder. Recuperado de https://www. ohchr.org/SP/ProfessionalInterest/Pages/ VictimsOfCrimeAndAbuseOfPower.aspx.

Olaya. C. (2015). Representaciones Sociales de Jóvenes Universitarios Frente al Conflicto Armado Colombiano. [Tesis de grado]. Universidad Santo Tomas, Colombia
Otálora, F. (2019). La violencia de origen criminal, articulación con la violencia política como factores de inestabilidad en el Catatumbo (20102018). Perspectivas en inteligencia, 11(20): 5169.

Peña, M. (2013). Daño Ambiental y prescripción. Revista Judicial, 109, 117-143.

Prieto, G. \& Delgado, A. (2010). Fiabilidad y validez. En: Revista Papeles del Psicólogo. Vol. 3, No. (1), p. 67-74.

Quiceno, F. L. (2013). Reconocimiento de beligerancia a grupos armados al margen de la ley como tránsito a una humanización de la guerra en Colombia. (A recognition of belligerency armed groups outside the law as a transit humanization war in Colombia). CES Derecho, 4(2), 91-100.

Ríos, J. (2016). Los enclaves de la violencia en Colombia, 1998 - 2012 [Tesis Doctoral] Universidad Complutense de Madrid.

Salazar, J. A. A., Barranco, L. A., Ruiz, L. K. J., Marín, M. P. R., \& González, L. R. (2016). Secuelas psicológicas de la guerra en mujeres forzadas a desplazarse. Revista internacional de psicología, 15(1), 2.

Toro. J. D. M. (2010). Acompañamiento psicosocial a niños y niñas víctimas del conflicto armado en Colombia: un escenario para la acción sin daño. [Tesis de grado]. Universidad Nacional de Colombia, Bogotá

Tovar. A. T. (2016). Inclusión educativa y desplazamiento forzado: una alternativa pedagógica desde las narrativas. [Tesis de grado]. Universidad Libre, Colombia

Unidad de Víctimas. (2020). Registro Único de Victimas. Recuperado de https://rni. unidadvictimas.gov.co/RUV 